\title{
SUR LA TRADUCTION DE LA POÉSIE DE MILOVAN DANOJLIĆ EN FRANÇAIS
}

\author{
UDC 821.163.41.09-1 Danojlić M. \\ $81 ' 255.2$
}

\section{Nataša Živić, Selena Stanković}

Université de Niš, Faculté de philosophie, Département de langue et littérature françaises, Niš, Serbie

\begin{abstract}
Résumé. Dans le présent travail, nous examinons la traduction française de quelques poèmes de Milovan Danojlić du recueil «Pesme » [Poèmes] (1980). Les poèmes étudiés sont traduits en français par Vesna Bernard-Radović et publiés dans le recueil « Seule la lumière : poèmes, 1957-2007 » (2010). Du point de vue morphosyntaxique, sémantique et stylistique, nous analysons la manière de transmettre l'idée de l'original et le style du poète et nous déterminons si la traductrice réussit à transposer dans la langue cible le rythme original et la rime du poème. Notre recherche s'effectue dans le cadre de la conception linguistique de la traduction et par le biais de la méthode contrastive. En vue d'explorer les transformations traductologiques faites lors du processus de traduction, nous nous servons de la classification des techniques proposée par Newmark (1988) et fondée sur la typologie établie par Vinay et Darbelnet (1972). Le corpus de notre recherche est composé de trois poèmes de Danojlić : "Insekti »/«Les insectes », «Peršun »/ Le persil » et «Dunja »/《L Le coing ».
\end{abstract}

Mots clés : traduction, poésie, technique traductologique, langue serbe, langue française.

\section{INTRODUCTION}

La traduction de la poésie impose au traducteur des preuves et des obstacles plus sérieux que ceux posés par la traduction de la prose. Durant ce processus, comme le remarque R. Konstantinović (1981, 119-139), outre les difficultés issues de la transmission du sens et des valeurs stylistiques, apparaissent des exigences provenant de la reproduction de la forme qui s'avère un segment extrêmement important dans l'œuvre poétique. Dans la poésie, la forme et le contenu constituent une unité dialectique - le contenu n'existe pas

Submitted July 1, 2019; Accepted September 9, 2019

Corresponding author: Selena Stanković

University of Niš, Faculty of Philosophy

E-mail: selena.stankovic@filfak.ni.ac.rs 
sans forme. Celle-ci est établie par le poète lors de sa création et elle est, en tant que modèle, offerte au traducteur qui doit, pour sa part, produire une nouvelle version du modèle. La tâche du traducteur est en même temps plus facile et plus délicate que celle de l'auteur de l'original : d'un côté, elle se montre plus simple car le traducteur n'a pas à penser à ce qu'il va écrire, mais, d'un autre côté, elle paraît plus complexe parce que le traducteur doit reproduire fidèlement le modèle créé. Le devoir primaire du traducteur est, en conséquence, de transférer adéquatement la forme de l'original. Le sens du poème se transmet plus tard. Vu que la traduction représente l'explication individuelle de l'original, le traducteur de la poésie, se trouvant confronté à une multitude de demandes, est souvent obligé de juger les éléments à préserver et les éléments à omettre dans sa version du modèle du poète. Il se décide, donc, pour une certaine hiérarchie des valeurs du texte original. Il cherche les solutions non au niveau du syntagme ou du vers, mais au plan du poème, car seul l'œuvre poétique, le poème en entier rend l'unité de traduction du poème. Si un poème est correctement expliqué, il sera traduit avec succès. L'objectif de la traduction de la poésie est, selon Konstantinović, d'inciter auprès du lecteur de la traduction l'impression identique ou au moins l'image similaire à celle que provoque le texte poétique original par rapport à son lecteur. Pour M. Danojlić (1981, 251), la meilleure traduction est celle dans laquelle le traducteur ne se voit pas et ne s'entend pas ; le traducteur de la poésie est, d'après notre poète et traducteur, un artisan.

B. Čović $(1994,125)$ conclut que tous les théoriciens de la traduction, tous les critiques et les traducteurs eux-mêmes se mettent d'accord avec l'idée que la traduction de la poésie représente la plus exigeante forme de la traduction artistique et que le genre lyrique, étant son domaine plus restreint, se trouve à la limite de l'impossible. De plus, c'est l'unique forme de la traduction qui porte à bon droit la valeur artistique. Elle diffère de toutes les autres formes par la difficulté essentielle : transposer la forme composant une harmonie unique avec le contenu poétique.

Dans cet article, nous examinons la traduction française de quelques poèmes de Milovan Danojlić du recueil Pesme [Poèmes] (1980). Les poèmes analysés sont traduits en français par Vesna Bernard-Radović ${ }^{1}$ et publiés dans le recueil Seule la lumière : poèmes, 1957-2007 (2010). Du point de vue morphosyntaxique, sémantique et stylistique, nous étudions la manière de transmettre l'idée de l'original et le style du poète et nous déterminons si la traductrice réussit à transposer dans la langue cible le rythme original et la rime du poème. Notre recherche s'effectue au sein de l'approche linguistique de la traduction et par l'intermédiaire de la méthode contrastive étroitement liée à la théorie de la traduction. Pour explorer les transformations traductologiques réalisées au cours du processus de traduction, nous nous servons de la classification des techniques proposée par Peter Newmark (Textbook of Translation, 1988) et basée sur la typologie de Jean-Paul Vinay et Jean Darbelnet (Stylistique comparée du français et de l'anglais, 1972). Le corpus de notre recherche comprend trois poèmes de Danojlić : Insekti (1980, 105)/Les insectes (2010, 55), Peršun $(1980,64) / L e$ persil $(2010,56)$ et Dunja $(1980,74) /$ Le coing $(2010,57)$.

\footnotetext{
${ }^{1}$ Vesna Bernard-Radović a traduit également des œuvres en prose de Milovan Danojlić, pour plus de détails voir Serbica, s.a.
} 


\section{SUR LA TRADUCTION DE LA POÉSIE}

Bien que la traduction de la poésie s'avère le plus excitant type de traduction, voire quelquefois un processus impossible, elle propose les principales directions de la pensée théorique sur la traduction en général (Sibinović 1990, 155). Pour de nombreux traducteurs, la traduction de la poésie représente le problème central de leur pensée. Ainsi, en expliquant de nombreuses approches explicatives de la traduction et en se focalisant sur l'approche poétologique, M. Guidère (2013, 52-55) renvoie particulièrement à E. Etkind qui, dans son ouvrage Un Art en crise (1982), déclare que la maladie de la traduction de la poésie est, en effet, la rationalisation systémique de l'original laquelle ignore l'unité indissociable du poème. Comme le précise Guidère, le théoricien russe regrette l'absence de véritable critique de la traduction telle que la critique des œuvres littéraires et il croit que si la traduction des vers subit aujourd'hui une grande crise, c'est à cause du manque de critique ; si la critique des traductions n'existe pas, les traductions trompent le lecteur. Selon Etkind, il existe deux grands mouvements dirigés par les plus grands poètes de la littérature française : Charles Baudelaire et Paul Valéry. D'après Baudelaire, il est impossible de traduire la poésie sans la traduire en prose rimée ; ce procédé ne donne non plus la forme parfaite, mais le plus grand mal est l'imitation rimée. Valéry estime toutefois qu'il ne suffit pas de traduire simplement le sens poétique, mais plutôt de tenter de transférer la forme, c'est-à-dire la prosodie de l'original. M. Guidère évoque, quant à lui, que si la fidélité repose uniquement sur le sens, cela sous-entend alors qu'il s'agit d'une sorte de tromperie.

Avant d'entrer dans le processus de traduction, le traducteur doit bien comprendre le poème original. Dans le poème, comme le signale $\mathrm{M}$. Stojnić $(1980,186)$, la frontière entre la forme et le contenu n'existe presque pas, c'est-à-dire que les structures formelles du poème ne sont qu'une autre forme d'expression du sens qu'il comporte. Ces structures formelles sont un signe plus universel, compris comme un élément musical transcendant les barrières linguistiques ; la sonorité transmet, donc, le sens même à l'auditeur qui ne comprend pas les signes linguistiques concrets. L'idéal à atteindre est la transmission de sonorité ainsi que de signification. Lors de la traduction, en se penchant sur leur liberté d'expression, les traducteurs changent parfois le message du texte d'origine, en l'altérant de cette manière ; les nuances et les variations résultant de la nature de la langue de traduction par rapport à la langue du texte original sont inévitables. C'est la raison pour laquelle il est nécessaire parfois de changer le sens du mot en vue d'obtenir la rime ou limiter le nombre de syllabes conformément à l'original. De même, S. Babić attire l'attention sur la dissemblance entre le contexte original et le contexte cible et trouve qu'en raison des différences culturelles et linguistiques, les traductions étant linguistiquement correctes s'avèrent néanmoins pauvres et mortes car elles ne sont pas susceptibles de subsister dans une nouvelle culture et une nouvelle langue (Babić 1986, 226-227).

Selon Stojnić $(1980,97)$, durant la traduction de la poésie, les figures stylistiques - la métaphore, la métonymie et la synecdoque - occupent une place particulière et importante. C'est pourquoi les images poétiques créées par ces figures ne sont pas de simples créations linguistiques. Les mots ne sont stylisés que dans leur contexte, par rapport à d'autres mots qui actualisent et concrétisent leur signification, seule et unique dans ce cas-là. Ensuite, l'auteure mentionne Y. Tynyanov qui, dans son ouvrage sur la problématique de langue des vers, explique que le mot est un caméléon changeant constamment non seulement les nuances, mais également les couleurs variées ; l'abstraction du mot représente une sorte de cercle rempli à chaque fois d'une nouvelle manière et dépendant de l'environnement lexical 
dans lequel il se trouve. Cette abstraction de mot est une sorte de coupe transversale de différentes structures lexicales et fonctionnelles. Enfin, M. Stojnić conclut que le traducteur n'a pas le droit de changer l'image poétique, même s'il l'est nécessaire pour que le rythme poétique ou bien la rime soient réalisés. L'image acoustique du poème ne peut pas être transférée dans son intégralité. Le matériel vocal du poème, la nature des sons de la langue d'origine, la nature des accents, les caractéristiques mélodiques et intonatives des syntagmes et des phrases ne sont pas dans la plupart des cas répétables dans une autre langue. En ce sens, la version traduite du poème ne s'approche que de sa version originale. Les significations décalées ne peuvent être présentes qu'en nuance dans la sélection de synonymes partiels, mais l'ensemble de l'image poétique ne doit pas être perturbé, ainsi que le message global du poème ne peut pas être nuancée.

B. Čović $(1986,36)$ démontre aussi que les problèmes du traducteur se multiplient lorsque la signification des expressions phraséologiques et idiomatiques, à cause de l'improvisation du poète, devient tellement déformée qu'il est difficile d'en reconnaître la source. R. Marojević (1988, 181) met l'accent sur le plan phono-rythmique de l'œuvre poétique, en tant que niveau d'analyse le plus élevé de la poétique de traduction ; ce plan est étroitement lié au plan lexico-sémantique ainsi qu'au plan des images poétiques. Le langage du poème, le symbolisme, la polysémie des mots et des structures grammaticales sont indissociables de l'ensemble et des rythmes qui s'entrelacent dans les poèmes. Toutes les couches d'une expression poétique doivent être intégrées au vers correspondant, à la strophe, au poème pour que l'orchestration des rimes soit dans l'harmonie avec le texte original. P. Newmark (1988, 163-166) souligne lui aussi l'importance des paroles dans la poésie où leurs propriétés portent une si grande importance, plus considérable que dans d'autres genres littéraires. Il ajoute également que si le mot est la première unité de signification, la phrase ou l'énoncé ne font pas la deuxième, mais c'est le vers qui représente l'unique double concentration d'unités. En outre, dans le texte, il est important de transférer parallèlement la ponctuation reproduisant le ton de l'original et la signification correspondante de métaphore. En parlant des métaphores, Newmark révèle que toutes les images possèdent une source universelle, culturelle et personnelle et que le traducteur ne doit faire aucune concession si ce n'est pour transférer la culture étrangère à son équivalent original. Il insiste sur le fait que la manière, le rythme, la métrique, le son et la structure dépendent du poème lui-même, mais aussi de la poétique individuelle du traducteur.

Outre par les auteurs mentionnés (Konstantinović 1981 ; Danojlić 1981 ; Čović 1994, 1986 ; Sibinović 1990 ; Guidère 2013 ; Stojnić 1980 ; Babić 1986 ; Marojević 1988 ; Newmark 1988), les aspects variés de la traduction de la poésie et les difficultés qui accompagnent le passage d'un texte poétique de l'original à la traduction sont examinés également par : G. Mounin (1963), J. Catford (1965), R. Jakobson (1966), L. Robel (1968), J. Levi (1982), V. Errante (1993), L. Venuti (1995), Y. Nakaji (2002), A. Mešonik (2004). Quant à la traduction de la lyrique du français vers le serbe et à l'inverse, nous trouvons les études de R. Konstantinović (2010) sur les sujets traductologiques aux niveaux stylistique et lexico-sémantique, sur les questions du contexte, etc., puis les textes de D. Janjić (2011) qui explore les problèmes de traduction en français des vers de J. J. Zmaj, de D. Matić, de V. Popa et des autres, ainsi que l'article de N. Krstić (2008) qui apporte l'analyse morphosyntaxique et lexico-sémantique de la traduction de V. Jovanović du poème L'Isolement d'A. de Lamartine. $^{2}$

\footnotetext{
${ }^{2}$ Pour ce qui est des analyses des traductions franco-serbes basées sur le classement des procédés proposé par P. Newmark, il est à renvoyer aux travaux de T. Đurin : Đurin 2014 et Đurin 2015. Les résultats de ces recherches
} 


\section{SUR LA POÉTIQUE DE MILOVAN DANOJLIĆ}

Comme l'explique notre poète Milovan Danojlić $(2004,5)$, la littérature pour enfants figure comme une possibilité d'expression littéraire, comme une aventure spirituelle qui ne s'est pas produite par hasard dans le monde moderne. Pour lui, le poème d'enfants est un phénomène qui dépasse d'étroites frontières littéraires et, d'une manière inattendue, affecte de nombreux domaines de la vie et de la culture. La vision la plus pittoresque de sa perception de la littérature d'enfants s'explique par les mots suivants : «Il apparut, dans ce monde, quelque chose de doux et de gai, facile et lumineux, insaisissable et imprévisible ; c'est un poème ou une apparition ; il apparaît ici ou rayonne là, il nous illumine et pénètre en nous, mais toujours et partout, nous rappelle la pureté basique de la vie, le fondement lointain du chant et de la pensée. Négligés et déviés, vaincus par les inquiétudes et les peurs, nous accueillons avec gratitude et confusion cette mélodie de la vie ancienne et oubliée. Mais quand elle finit son chant, quand elle se tait, nous regardons faiblement autour de nous dans le désir d'en saisir la source, de prévoir sa suite $»^{3}$ (Danojlić 1967, 114).

Ce grand poète crée des effets artistiques suggestifs et puissants déjà avec son premier recueil de poèmes pour enfants intitulé Kako spavaju tramvaji [Comment les tramways dorment] (1959). Venu de son village natal dans une grande ville, il a découvert la vie dans un environnement urbain où les tramways sont utilisés quotidiennement. L'objet de son poème est annoncé dans le titre du recueil pour approcher aux enfants ce moyen de transport qu'ils utilisent tous les jours sans y penser, en les incitant ainsi à y réfléchir. Dans ses œuvres poétiques, Danojlić peint les entourages rural et urbain ou symboliquement l'herbe, d'une part, et l'asphalte, de l'autre. Les particularités de ses poèmes sont le réalisme poétique, la symbolique des formes de la nature, l'impression fraîche. Son expression poétique s'incarne dans des visions matérielles presque tangibles et représente une congruence de relation vivante du poète vers le monde. Dans de nombreux poèmes sur les plantes, les fruits, les légumes, sur le monde animé, naturel, visible, M. Danojlić décrit son attachement à la terre et à la nature, son inclination profonde à la vie simple et conforme aux règles de la nature, ses penchants sincères à la bonté, à la générosité, aux valeurs familiales et aux qualités humaines en général. Dans l'ouvrage de Danojlić, les nombreux fruits et plantes sont anthropomorphisés et à travers cet univers l'on sent l'esprit de la vie intense dans la nature, ainsi que l'intégrité symbolique de l'humanité et de la nature. L'impression est que le poète se souvient de son enfance et des rassemblements après les semis ou les récoltes, où autour du chaud nid familial, on exprime la gratitude envers la terre pour les fruits qu'elle offre. Par leur rythme et leur mélodie, ses poèmes évoquent de vieilles mélodies éternelles. Le symbolisme de la beauté mythique que comportent les poèmes sur les dons magnifiques de la nature aide le poète à établir de nouvelles relations entre l'homme et la nature (Obradović 2005, 403-412). La forte liaison à l'univers naturel est, d'ailleurs, bien illustrée dans les trois poèmes que nous avons choisis pour notre étude traductologique.

éclaircissent avec succès l'emploi des interventions traductologiques et leur rôle dans la transmission du texte et du sens de la langue de départ à la langue d'arrivée.

${ }^{3}$ Traduit du serbe en français par les auteures de l'article. 


\section{ANALYSE DU CORPUS}

Le théoricien britannique P. Newmark est le partisan de la traduction littérale, soit qu'il s'agisse d'une traduction littéraire ou non littéraire. Le produit de ce type de traduction est la traduction littérale et fidèle ('close translation'). Au cas où la traduction littérale ne produit pas l'effet souhaité, il faut accéder aux transformations traductologiques qui sous-entendent l'usage des techniques traductologiques, telles que (Newmark 1988, 45-53, 58, 81-91, 114 $124,163-170 ; 1991,3)$ : a) l'emprunt incluant la transcription et la translittération ; b) la naturalisation ; c) l'équivalence (l'équivalent culturel, l'équivalent fonctionnel) ; d) le synonyme ; e) le calque ; f) la transposition ; g) la modulation ; h) l'analyse composante ; i) la réduction ; j) l'expansion ; k) la paraphrase ; l) l'adaptation ; m) la combinaison des procédés. Pour les auteurs J.-P. Vinay et J. Darbelnet (1972, 46-55), la traduction littérale est, à côté du calque et de l'emprunt, la traduction directe, alors que les autres sont obliques.

L'emprunt se définit en tant qu'emploi d'un terme de la langue de départ sans le traduire ; c'est la technique utilisée en particulier pour les noms propres, les toponymes, les noms de journaux et de périodiques, les appellations des institutions publiques et privées, les termes culturels, les appellations des rues, etc. Le procédé de naturalisation comprend l'adaptation phonétique et morphologique d'un mot à la structure de la langue d'arrivée. L'équivalence est la transformation qui restitue le même contexte linguistique en recourant aux moyens entièrement différents. Ainsi, l'équivalent culturel signifie le remplacement d'un terme culturel original par un terme culturel de la langue d'arrivée. L'équivalent fonctionnel implique la traduction de mots liés à la culture de l'original, mais cette fois-ci, ces mots sont transmis par un nouveau terme spécifique qui neutralise ou généralise parfois la signification du mot de l'original. Le synonyme explique la proximité entre l'équivalent cible et le mot source du contexte et il est utilisé lorsqu'il n'existe pas d'équivalent formel suffisamment clair ou lorsqu'il s'agit d'adjectifs qualificatifs ou d'adverbes qui ne constituent pas des éléments clés de la structure syntaxique de la phrase. La technique de calque sous-entend l'emprunt d'un groupe de mots étranger avec la traduction littérale de ses constituants. La transposition se détermine comme le changement de catégorie grammaticale d'une unité sémantique ; il existe quatre modèles de transposition. La modulation désigne la variation faite par le changement de point de vue, de perspective et, très souvent, de catégorie de pensée. L'analyse composante consiste à séparer l'unité lexicale en deux ou trois composants ou même plus. Le procédé de réduction représente, en effet, l'omission de certains éléments du texte original sans nuire au sens du message. La technique inverse est l'expansion qui repose sur l'augmentation de volume de l'unité de traduction par ajout de nouveaux éléments. La paraphrase dénote l'explication du contenu sémantique de la langue de départ et, en général, cette transformation entraine l'emploi de plus de mots pour manifester l'idée originale. Le procédé d'adaptation suppose l'emploi d'un équivalent reconnu entre les deux contextes linguistiques et culturels. Le processus de traduction connaît également les combinaisons diverses des techniques dans le but de créer une traduction fidèle.

Notre examen, s'appuyant sur le classement des procédés traductologiques précité, s'effectue sur le texte du poème Insekti [Les insectes] faisant partie du chapitre Pesme o zdravom životu [Les poèmes sur la vie saine] du recueil Pesme (Danojlić 1980, 105) et sur les vers de deux poèmes du bloc thématique Rodna godina [L'année féconde] du même recueil : Peršun (Ibid, 64) et Dunja (Ibid, 74). Le corpus parallèle est constitué de leurs traductions françaises parues dans la publication Seule la lumière : poèmes, 1957-2007 : Les insectes (Danojlić 2010, 55), Le persil (Ibid, 56) et Le coing (Ibid, 57). 


\subsection{Insekti/Les insectes}

Kapljice zejtina: po ivici

Šume miču se klizavi svici, -

Zvezdani insekti, punoglavi,

Petrolejske suze u travi;

Popac, na stazi, tu negde, sleva,

Naizmence sa sobom peva;

Dok roj komaraca iznad mog doma

Lebdi kao nacrt atoma.

\author{
Gouttes d'huile : à l'orée \\ Du bois, des lucioles dorées - \\ Insectes stellaires, pleins de superbe, \\ Larmes de pétrole dans l'herbe ; \\ Un grillon égaré sur la sente \\ En duo avec lui-même chante ; \\ Une nuée de moustiques sous le dôme \\ Dessine le schéma de l'atome.
}

Comme le montre notre analyse, la forme poétique originale est gardée dans la langue cible : une strophe composée de huit vers. Quant à la ponctuation, le constituant important du style qui est capable de devenir le discours poétique particulier (Čović 1994, 136-139) ${ }^{4}$, la traductrice réussit en général de préserver celle du poète. Le vers libre de l'original est conservé dans la traduction, en respectant la rime en pair. À l'intérieur du premier vers, le nom au pluriel Kapljice a été traduit par un substantif presque synonymique, mis également au pluriel : Gouttes ; toutefois, la signification diminutive de l'original n'a pas été transmise. L'enjambement du premier vers au deuxième po ivici/Šume est transféré littéralement et adéquatement à l'aide du syntagme l'orée/Du bois. Dans la traduction française de la structure phrastique miču se klizavi svici - /Zvezdani insekti, punoglavi,/Petrolejske suze u travi, nous remarquons l'application de plusieurs procédés traductologiques. D'abord, le prédicat miču se de l'original est par la réduction omis dans la traduction. Puis, le syntagme nominal klizavi svici devient le groupe français des lucioles dorées au moyen de trois techniques : la transposition (le changement de place de l'adjectif par rapport au nom), la modulation (le renversement de point de vue) et l'expansion (l'ajout de l'article). Ensuite, l'union nominale Zvezdani insekti se transforme en syntagme adéquat Insectes stellaires, mais avec l'ordre des mots inhérent au français (le nom + l'adjectif qualificatif), ce qui démontre l'emploi du procédé de la transposition. Enfin, l'adjectif apposé punoglavi de la langue de départ est par la modulation transmis en groupe adjectival pleins de superbe (la substitution du concret à l'abstrait).

Dans le quatrième vers, le groupe substantival serbe Petrolejske suze se traduit par la construction nominale Larmes de pétrole transmettant littéralement le sens ; ici, la traductrice se sert à bon droit de la transposition. Le complément circonstanciel de lieu $u$ travi est traduit littéralement - dans l'herbe - avec l'ordre des mots gardé et l'application de l'expansion (l'ajout de l'article défini).

Grâce à l'expansion et à la modulation, la traductrice peint l'atmosphère du vers suivant conformément à l'original : l'équivalent français du substantif serbe sujet Popac contient l'article indéfini - Un grillon -, alors que le syntagme prépositionnel na stazi, étant en fonction du complément circonstanciel de lieu, garde la forme syntaxique en recevant l'article défini - sur la sente. La suite de mots serbe tu negde, sleva devient par la modulation (la modification de perspective) l'adjectif français égaré. Dans la version française du vers, le groupe sur la sente occupe la dernière place et, avec le vers suivant, le sixième, fait partie d'une rime en pair (sente/chante); il exerce, en conséquence, la même fonction que l'adverbe sleva de l'original, rimé avec la forme verbale peva du sixième vers (sleva/peva). Le sixième vers contient le prédicat du vers précédent, Naizmence sa sobom

\footnotetext{
${ }^{4}$ Sur l'importance de la ponctuation en tant qu'élément du style de l'écrivain et sur la fidélité de sa transmission dans la traduction voir dans Čović 1994, 136-145.
} 
peva. Sa version française En duo avec lui-même chante tient l'ordre des mots de l'original serbe, mais avec une petite transformation : au moyen de la transposition, l'adverbe Naizmence obtient, pour son équivalent de traduction, la construction prépositionnelle $-E n$ duo.

Puis, afin de traduire les septième et huitième vers, la traductrice utilise la transposition : la subordonné temporelle Dok roj komaraca iznad mog doma/Lebdi kao nacrt atoma est modifiée en proposition indépendante juxtaposée par rapport à la précédente - Une nuée de moustiques sous le dôme/Dessine le schéma de l'atome. Nous constatons, dès lors, (a) la réduction de l'équivalent du subordonnant temporel serbe et (b) la traduction littérale du syntagme substantival roj komaraca avec l'intervention de l'expansion (l'addition de l'article indéfini) : Une nuée de moustiques. Dans le complément circonstanciel de lieu sous le dôme, en changeant la perspective la traductrice se sert de la modulation ; de fait, elle recourt à l'antonyme de la préposition serbe (iznad - sous) et à l'unité homophone du nom serbe (dom - dôme). Nous supposons que dans ce cas-là, la traductrice, motivée par ses affinités artistiques, reconnaît métaphoriquement le dôme comme l'abri, le domicile, la maison et, de plus, elle se décide à cette solution traductologique en raison de l'accord avec le mot suivant (atome).

Enfin, au sein du vers final du poème Lebdi kao nacrt atoma - Dessine le schéma de l'atome, nous apercevons le changement de point de vue et, en conséquence, la réorganisation traductologique à travers la modulation. Avec cette transformation astucieuse, la traductrice transmet fidèlement l'esprit et le coloris de l'original.

Notre investigation des corpus original et traduit découvre également une particularité stylistique du poème : l'emploi de la figure de l'allitération ${ }^{5}$. Dans le poème de Danojlić, il s'agit de la répétition de la consonne non-voisée - l'affriquée $c$ [ts], tandis que dans la traduction de Bernard-Radović, il est question de l'allitération de la consonne voisée - la liquide $l$ [1]. Nous concluons que la traductrice a trouvé, donc, une bonne solution pour donner une image sonore au vers et au poème, comme l'a fait l'auteur de l'original. Les mots contenant les consonnes répétées s'opposent à d'autres mots par leur texture sonore et, de cette façon, ils obtiennent une position importante dans la structure de motif du poème.

\subsection{Peršun/Le persil}

Kada zagrizem list peř̌una,

Duša mi postane puna, prepuna.

- Briznu, iz reckave ove travčice, Mirisne tačkice, slane varnice...

Od te plemenite mirođije

Život čistiji ukus dobije.

To struje iz žila, iz dubina,

Zeleni žmarci ce vitamina;

Gricneš ga: s vrhom se jezika slije Listić električne baterije.
Quand je mords dans une feuille de persil D’allégresse mon cour se remplit,

- Jaillissent de la sainte cette herbette Étincelles iodées, de suaves bluettes...

Avec cette noble odoriférante

La vie s'épanouit et s'enchante.

Circulent là, vivaces, épicés, Les verts frissons de vitamine $C$;

Tu mords et le bout de la langue pique Une foliole de pile électrique.

\footnotetext{
${ }^{5}$ Pour plus de détails sur l'allitération voir dans Simić 2001, 258.
} 
D'après notre examen de la traduction française du poème, la rime en pair de l'original est conservée, elle est restée fidèle au vers libre et à la structure formelle du poème original : cinq strophes de diptyque. La traductrice s'efforce le plus souvent de maintenir la ponctuation de M. Danojlić ; quelquefois il y a des variations, cependant, les qualités stylistiques et expressives de l'original sont transmises. En ce qui concerne la première partie de la première strophe Kada zagrizem list peršuna, la traduction adéquate et réussie se réalise par le biais du procédé de la traduction littérale, avec des changements conformément à la nature de la langue cible. Tout d'abord, il s'agit de l'expansion effectuée par l'ajout du pronom personnel je, et ceci suivant la nature morphosyntaxique du français qui exige le pronom sujet en tant que désinence verbale antéposée au verbe. Puis, le syntagme verbal subit la transposition étant donné que V. Bernard-Radović opte pour l'emploi transitif indirect du verbe mordre - (je) mords dans. Le complément d'objet serbe list peř̌una se transforme en construction française une feuille de persil qui préserve entièrement le caractère syntaxique (l'union nominale) et le sémantisme (la possession inaliénable de type partie-tout) de l'original en s'amplifiant grâce à l'expansion justifiée (l'article indéfini). Le deuxième vers de la même strophe en français D'allégresse mon cœur se remplit nous témoigne la combinaison habile des techniques : le travail de traduction a réuni la modulation, la transposition et l'expansion. Les intentions stylistiques et référentielles du vers original sont efficacement transmises à l'aide de la modulation : la traductrice réalise la substitution d'une partie à une autre partie (cœur - duša). Ensuite, par l'intermédiaire d'une transposition subtile, (a) le syntagme nominal serbe à datif possessif Duša mi (la possessivité inaliénable, la relation partie-tout) trouve son équivalent sémantique dans l'union substantivale à déterminant possessif mon cœur, tandis que (b) le prédicat postane puna, prepuna se convertit en verbe pronominal se remplit recevant une expansion moyennant le complément indirect D'allégresse. L'emploi de ce syntagme prépositionnel est motivé par l'idée de la gaieté présente dans le vers en serbe. La rime originale peršuna/prepuna est reformulée dans la traduction par persil/remplit.

L'analyse de la deuxième strophe démontre que la traductrice parvient à présenter au lecteur français la coloration émotionnelle et le spectre visuel de ces deux vers originaux. Ainsi, l'ordre des mots du premier vers de la deuxième strophe suit absolument et avec succès le texte original. La traduction littérale adéquate donne également la forme verbale Jaillissent en tant qu'équivalent du prédicat serbe Briznu. Par le biais de la modulation (le remplacement du concret par l'abstrait), l'adjectif reckava se transforme en adjectif français sainte. La justification d'un tel choix repose sans doute sur le fait que le persil, comme une plante, symbolise l'énergie vitale et l'énergie solaire, l'unité élémentaire de la vie, la naissance perpétuelle et le développement. Herbe symbolisant tout ce qui est curatif et revivifiant, le persil représente une manifestation de vie et porte ses vertus médicinales, il nous donne la santé, la vigueur et la fécondité (Chevalier et Gheerbrant 1982, 498, 764, 996) et c'est la raison pour laquelle il est dans la traduction de Bernard-Radović une sainte herbe. La traduction littérale caractérise également le transfert réussi du diminutif travčica du texte de départ au texte d'arrivée : herbette. Deux groupes nominaux juxtaposés du deuxième vers ont une distribution opposée en version originale et en version traduite : Mirisne tačkice, slane varnice - Étincelles iodées, de suaves bluettes ; l'adjectif synonymique iodées au lieu de salées révèle l'utilisation de la modulation (le remplacement de l'effet par la cause); au sein du syntagme de suaves bluettes, nous notons l'intervention de l'expansion conforme aux exigences grammaticales du français (l'article partitif). 
L'odeur délicieuse et la saveur douce de la vie, que Danojlić laisse développer dans le troisième segment de son poème, la traductrice interprète en harmonisant la modulation et la paraphrase. Elle recourt à la substitution du concret à l'abstrait et se sert de l'adjectif substantivé odoriférante à la place du nom original mirođija. Le vers suivant devient paraphrasé en gardant le terme clé et le contenu sémantique qui manifestent la beauté de l'univers naturel et la joie de vivre.

Afin de présenter la tonalité de la quatrième strophe de l'original - l'ardeur du persil, l'herbe puissante qui conserve et rend la santé - la traductrice applique simultanément la modulation par le changement de point de vue (To struje iz žila, iz dubina - Circulent là, vivaces, épicés) et la traduction littérale (Zeleni žmarci ce vitamina - Les verts frissons de vitamine $C$ ) combinée avec l'expansion suivant la nature de la langue d'arrivée (l'article défini).

Dans la traduction de V. Bernard-Radović, l'atmosphère de la dernière strophe de $\mathrm{M}$. Danojlić se transmet par un assemblage raffiné des procédés traductologiques. Ainsi, à l'intérieur du premier vers, nous voyons la modulation basée sur le renversement de point de vue : $s$ vrhom se jezika slije - et le bout de la langue pique. Outre la technique mentionnée, ici agissent en même temps la réduction (l'omission du personnel complément direct) et l'expansion (l'usage obligatoire du personnel sujet) : Gricneš ga - Tu mords. La réorganisation de la structure du premier vers a entraîné une ponctuation différente par rapport à celle de l'original ; pourtant elle a gardé la valeur stylistique originale et a permis la rime avec le vers suivant pique/électrique en tant qu'équivalent de la rime de l'original serbe slije/baterije. Le dernier vers du poème se reformule en français d'abord par l'expansion : Listić - une foliole. Ensuite, la traductrice utilise la transposition fondée sur le changement de position de l'adjectif par rapport au substantif : električne baterije - de pile électrique.

\subsection{Dunja/Le coing}

Kô svetiljka koja tinja i kunja, mutnim se sjajem preliva dunja; taj sjaj: sve mesečine u nj se sliše, sjaj, al' i miris, jošte više, koji nas, uvek iznova, seća da negde - van zemlje - postoji sreća, i da smo, pre no što smo na zemlju pali, sve već videli i sve znali.

\author{
Comme une veilleuse qui cligne et somnole, \\ d'une rêveuse clarté le coing s'auréole ; \\ cette clarté : tous les clairs de lune y dansent, \\ la clarté mais plus encore la fragrance, \\ toujours elle nous rappelle que, ailleurs, \\ quelque part, hors de terre, il y a le bonheur, \\ et que, avant d'être sur la terre chus, \\ nous avions déjà tout vu, tout connu.
}

L'étude de la traduction française du poème de M. Danojlić Dunja/Le coing fait voir que V. Bernard-Radović cherche à conserver la structure formelle (une seule phrase) et la rime en pair imposées par le modèle. La ponctuation originale est maintenue en proportion des règles de la ponctuation au sein de la langue française ; grâce à cela, la traductrice réussit donc de communiquer le rythme et la mélodie des vers originaux et de garder l'élément important de la poétique individuelle de l'auteur du modèle. Au moyen de la traduction littérale, l'organisation structurelle du premier vers devient identique à celle de l'original : Kô svetiljka koja tinja i kunja-Comme une veilleuse qui cligne et somnole, avec le soutien de l'expansion - l'ajout de l'article indéfini une.

La traduction adéquate du deuxième vers se caractérise premièrement par l'emploi de la modulation : à l'aide de la suppléance du concret par l'abstrait, apparaît l'adjectif rêveuse comme équivalent de traduction à l'adjectif original mutnim. Deuxièmement, le syntagme d'instrumental mutnim se sjajem - d'une rêveuse clarté subit l'expansion en recevant 
l'article indéfini. Troisièmement, la transposition change la distribution sujet + prédicat : preliva dunja - le coing s'auréole, la structure où, du reste, se manifeste une nouvelle intervention de l'expansion selon la nature du français (l'article défini) ; notre hypothèse est que Bernard-Radović tâche de rimer le verbe avec la forme verbale du premier vers. Enfin, pour transmettre le prédicat, la traductrice utilise la modulation en changeant de point de vue : preliva - s'auréole.

La traduction littérale qualifie dans la plus grande partie le troisième vers : taj sjaj: sve mesečine u nj se sliše - cette clarté : tous les clairs de lune y dansent. Outre cette technique, nous notons également (a) l'expansion qui élargit le groupe substantival par l'article défini, (b) le type de transposition par lequel une structure/forme grammaticale de la langue de départ se remplace par une autre équivalente de la langue d'arrivée : mesečine - clairs de lune (le substantif et le groupe substantival), se sliše - dansent (l'aoriste et le présent de l'indicatif), $u n j-y$ la transposition qui apporte (le pronom adverbial et le syntagme prépositionnel), et (c) la modulation sensorielle pour transmettre la forme verbale et l'intention référentielle du poète.

Bien que la traductrice varie la ponctuation du quatrième vers par rapport à l'original de Danojlić (sjaj, al' i miris, jošte više - la clarté mais plus encore la fragrance), la traduction reste adéquate et littérale. Ici, nous remarquons la double expansion (deux articles définis) et une différente distribution des éléments afin de créer la rime avec le vers précédent (sliše/više - dansent/fragrance). Le coloris situationnel de l'original est entièrement transmis dans le vers français. Au surplus, dans la reprise consécutive de l'unité syntaxique homoforme au sein de deux derniers vers - taj sjaj/sjaj-, nous apercevons la présence de la répétition d'épizeuxe (Kovačević 2015, 325-326) fidèlement transmise dans la traduction française - cette clartélla clarté ; au moyen de ce procédé stylistique d'intensification, le poète et la traductrice souligne le terme (sjaj/clarté) qui assume l'accent logique et emphatique de l'énoncé et devient son membre le plus marqué au niveau communicatif.

À l'aide de la transposition, la relative serbe du vers suivant est restructurée en proposition principale française de manière à présenter correctement l'idée et l'atmosphère de l'original. Simultanément avec ce procédé, se déroulent la réduction (uvek iznova toujours) et l'expansion par l'usage, en position finale, de l'adverbe ailleurs qui annonce le début du vers suivant et qui est bien rimé avec sa fin : ailleurs/bonheur comme équivalent à la rime de l'originale sećalsreća.

Pour ce qui est du sixième vers, la traductrice se sert de la traduction littérale et parvient à reproduire le sens et l'ambiance du modèle, ainsi que le style de son auteur. À l'intérieur du réseau de ponctuation, notre analyse démontre que $M$. Danojlić utilise le tiret en vue d'indiquer plus fort un élément de la phrase (van zemlje) ; cependant V. Bernard-Radović opte pour la virgule en tant que substitut du tiret en perdant dans une certaine mesure l'effet d'insistance sur le membre concerné (hors de terre) ; les deux signes graphiques se comportent de la même manière en deux langues observées (Pešikan, Jerković, Pižurica 2010, 104, 122-125 ; Kannas 2012, 61). Dans le même vers, nous remarquons l'application réussie de la transposition : la structure impersonnelle française il y a remplace le prédicat postoji et permet que son complément le bonheur garde la position finale de la même façon que son équivalent serbe - le sujet sreća ; la rime est également préservée (seća/sreća ailleurs/bonheur).

La reproduction du vers suivant $i$ da smo, pre no što smo na zemlju pali-et que, avant d'être sur la terre chus se déroule dans les limites de la transposition en respectant la distribution des éléments phrastiques. Les intentions référentielles et expressives de l'original sont présentées d'une manière adéquate dans la traduction. 
Enfin, le dernier vers du poème subit l'expansion déclenchée par la syntaxe du français - l'usage du pronom personnel sujet nous - et la réduction motivée par le nombre des syllabes du vers - l'omission de la conjonction de coordination. Nous notons aussi la réorganisation de l'ordre des mots au sein du vers d'après la nature de la langue cible.

Dans le poème examiné, pour transférer la sonorité de l'image poétique originale, la traductrice V. Bernard-Radović se sert de la figure stylistique de l'allitération en suivant, du reste, le caractère du modèle. Ainsi, l'allitération originale des sonantes palatales $n j[n]$ et $l j$ [lj], et ceci depuis le titre, et des consonnes palatales non-voisées - la répétition de

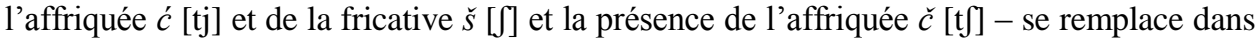
la traduction par des liquides françaises $l[1]$ et $r$ [r], les sonantes orales et voisées. Dès lors, nous pouvons dire que la traduction française du poème de $M$. Danojlić assume sa fonction phonétique par rapport à son modèle : elle reproduit l'effet mélodique et rythmique bien adapté au contexte linguistique et aux intentions poétiques. L'allitération utilisée contribue au renforcement communicatif de certains membres du texte.

\section{CONCLUSIONS}

Notre analyse traductologique et contrastive des poèmes Insekti/Les insectes, Peršun/Le persil et Dunja/Le coing démontre que le sens et la forme des œuvres poétiques de Milovan Danojlić ont été traduits en français d'une manière adéquate et précise. La traductrice Vesna Bernard-Radović réussit à récréer pour les lecteurs français le style du poète serbe et les qualités linguistiques et poétiques qui ornent l'original. Sa traduction s'avère fidèle en même temps au texte de départ, à ses motifs et à sa structure formelle, ainsi qu'à l'esprit de la langue d'arrivée. Elle revit avec succès le contexte situationnel, la puissance de la nature et le coloris visuel de l'univers présenté dans les vers du poète serbe. Les odeurs et les saveurs enivrantes de nature, les beautés de la vie simple et saine, la joie de vivre et le bonheur sont les motifs et les messages des poèmes que la traduction française transfère soigneusement et habilement. En combinant, d'une part, les moyens morphosyntaxiques du français, les valeurs sémantiques des lexèmes et des outils stylistiques avec les procédés traductologiques, d'autre part, l'auteur de la traduction parvient à transférer les intentions référentielles, expressives et émotionnelles de l'original et transmet efficacement les particularités linguistiques et stylistiques du poète et de ses poèmes.

Selon les résultats de notre investigation, la traductrice préserve la forme des poèmes analysés en gardant le vers libre du modèle, son nombre de vers et sa rime en pair, elle respecte le procédé métrique de l'enjambement du modèle ce qui prouve sa maitrise de versification. Dans le but d'illustrer pleinement le rythme et la mélodie du texte original, elle cherche à maintenir le lacis original de ponctuation, étant l'élément important de la poétique individuelle d'un poète ; les signes de ponctuation se modifient au cas où les règles de la langue cible le demandent. V. Bernard-Radović se sert astucieusement de la traduction littérale, en tant que procédé direct, mais également des techniques traductologiques suivantes :

a) l'expansion, dont la fréquence est la plus haute ce qui provient de la différence structurale entre les deux langues contrastées. Il est question de l'ajout des articles indéfini et défini particulièrement, mais aussi bien de l'article partitif ; apparaît également le pronom personnel sujet dont l'emploi est conditionné par les règles grammaticales du français ;

b) la modulation, aussi fréquente que l'expansion, s'utilise dans plusieurs modèles - le renversement de point de vue, la substitution du concret à l'abstrait, le remplacement d'une partie à une autre partie et de l'effet par la cause, la modulation sensorielle ; 
c) la transposition, très présente parmi les interventions traductologiques dans notre corpus, apparaît parallèlement à deux techniques précédentes. Pour ce qui est de l'aspect de transposition, nous notons les premier et troisième types. Le premier comprend le changement de position de l'adjectif par rapport au substantif. Le troisième est le type qui sous-entend le remplacement de la forme/structure de la langue de départ par une autre construction se montrant plus appropriée dans la langue d'arrivée ; à ce niveau, nous rencontrons les transformations à l'intérieur du syntagme nominal et du groupe verbal, au plan de la construction phrastique et dans l'union du sujet et du prédicat ;

d) la réduction s'emploie dans notre corpus beaucoup plus rarement que les trois techniques précédentes, mais elle apporte de bonnes solutions traductologiques. Ainsi, la traductrice omet : l'expression du prédicat et du subordonnant temporel, l'utilisation du personnel en fonction du complément d'objet direct, l'usage de l'adverbe ;

e) la paraphrase, la transformation à laquelle la traductrice recourt seulement deux fois, mais qui donne la traduction réussie.

Notre recherche du point de vue stylistique révèle que pour présenter l'image sonore de l'original, la traductrice Bernard-Radović utilise simultanément avec M. Danojlić la figure de style de l'allitération. De même, reproduisant l'accent sémantique et emphatique de l'original et son but communicatif, la traductrice se sert, d'une manière identique au poète, du procédé stylistique d'intensification d'épizeuxe.

Ce travail contribue à deux projets scientifiques : "Les langues, les littératures et les cultures romanes et slaves en contact et en divergence "( $\left.N^{o} 81 / 1-17-8-01\right)$, financé par la Faculté de philosophie de l'Université de Niš, l'Agence universitaire de la francophonie et l'Ambassade de France en Serbie, et "La traduction dans le système de la recherche comparée des littératures et cultures serbe et étrangères » ( $N^{o}$ 178019), financé par le Ministère de l'Éducation, de la Science et du Développement technologique de Serbie.

Les auteures de cet article remercient vivement Sanja Bošković-Danojlić, Maître de conférences-HDR à l'Université de Poitiers, qui a eu l'amabilité de leur procurer le recueil des poèmes de Milovan Danojlić dans la traduction française. Sans son aide précieuse, il aurait été très difficile d'achever cette recherche.

\section{RÉFÉRENCES}

Babić, Sava. Razabrati u pletivu: Eseji o prevodilačkom činu. Novi Sad, Književna zajednica Novog Sada, 1986. Catford, John Cunnison. A Linguistic Theory of Translation. London, Oxford University Press, 1965.

Chevalier, Jean et Alain Gheerbrant. Dictionnaire des symboles. Paris, Éditions Robert Laffont S.A. et Éditions Jupiter, 1982.

Čović, Branimir. Umetnost prevođenja ili zanat. Novi Sad, Književna zajednica Novog Sada, 1986.

Čović, Branimir. Poetika književnog prevođenja. Beograd, Naučna knjiga, 1994.

Danojlić, Milovan. Lirske rasprave. Novi Sad, Matica srpska, 1967.

Danojlić, Milovan. „Pesnik kao prevodilac.” U Teorija i poetika prevođenja. Priredio Ljubiša Rajić. 243-260. Beograd, Prosveta, 1981.

Danojlić, Milovan. Naivna pesma: ogledi i zapisi o dečjoj književnosti. Beograd, Zavod za udžbenike i nastavna sredstva, 2004

Đurin, Tatjana. «Les explications du traducteur : trahison ou renaissance de l'œuvre originale ? », dans Јазични и културни проникнувања низ преведувањето и толкувањето, éd. par Т. Панова-Игњатовиќ. Скопје, Филолошки факултет „Блаже Конески”. 2015, p. 149-158.

Đurin, Tatjana. « Gargantuino mladovanje i slobodna doslovnost Stanislava Vinavera », dans Jezici i kulture u vremenu i prostoru III, éd. par S Gudurić, M. Stefanović. Novi Sad, Filozofski fakultet u Novom Sadu, 2014, p. 325-332.

Errante, Vincenzo. La traduzione di poesia ieri e oggi. Milano, Cisalpino, 1993. 
Guidère, Mathieu. Introduction à la traduction. Bruxelles, De Boeck, 2013.

Jakobson, Roman. Lingvistika i poetika. Choix M. Ivić, S. Marić. Traduit par D. Pervaz et al. Beograd, Nolit, 1966. Janjić, Dušan. Književna preplitanja: Ogledi i zapisi o francusko-srpskim književnim vezama. Beograd, Altera, 2011. Kannas, Claude. Bescherelle: L'orthographe pour tous. Paris, Hatier, 2012.

Konstantinović, Radivoje. «O prevođenju poezije », dans Teorija i poetika prevođenja. Choix Lj. Rajić. Beograd, Prosveta, 1981, p. 119-139.

Konstantinović, Radivoje. O prevođenju poezije i drugi ogledi. Novi Sad, Adresa, 2010.

Kovačević, Miloš. Stilistika i gramatika stilskih figura. IV bitno dopunjeno izdanje. Beograd, JASEN, 2015.

Krstić, Nenad. "Lamartinova Osama u prevodu Vladimira M. Jovanovića », dans Zbornik Matice srpske za književnost i jezik 56 (1), 2008, p. 25-37.

Levi, Jirži. Umjetnost prevođenja. Traduit par B. L. Dabić. Sarajevo, Svjetlost, 1982.

Marojević, Radmilo. Lingvistika i poetika prevođenja. Beograd, Naučna knjiga, 1988.

Mešonik, Anri. Od lingvistike prevođenja do poetike prevođenja. Traduit par B. Anđelković, Z. Đaković. Beograd, Rad-Alternativna akademska obrazovna mreža, 2004.

Mounin, Georges. Les problèmes théoriques de la traduction. Paris, Gallimard, 1963.

Nakaji, Yoshikazu. « L'œuvre poétique entre traduction et création ». Littérature 125, L'œuvre illimitée, 2002, p. 66-72.

Newmark, Peter. Textbook of Translation. New York, Prentice Hall International ELT, 1988.

Newmark, Peter. About Translation. United Kingdom, Multilingual Matters, 1991.

Obradović, Slavoljub. Književnost za decu. Aleksinac, Viša škola za obrazovanje vaspitača, 2005.

Pešikan, Mitar, Jovan Jerković et Mato Pižurica. Pravopis srpskoga jezika. Novi Sad, Matica srpska, 2010.

Robel, Léon. " Problèmes théoriques de la traduction de la poésie russe en français. » Revue des Études Slaves 47(1-4), 1968, p. 123-128.

Serbica, s.a. « Serbica, le portail de littérature serbe en langue française ». Accédé le 22 mai 2019. https://serbica.ubordeaux-montaigne.fr/index.php/d-22/63-danojli-danoilitch-milovan.

Sibinović, Miodrag. Novi original: uvod u prevođenje. Beograd, Naučna knjiga, 1990.

Simić, Radoje. Opšta stilistika. II izdanje. Beograd-Nikšić, JASEN, 2001.

Stojnić, Mila. O prevođenju književnog teksta. Sarajevo, Svjetlost, 1980.

Venuti, Laurence. The Translator's Invisibility. London and New York, Routledge, 1995.

Vinay, Jean-Paul et Jean Darbelnet. Stylistique comparée du français et de l'anglais. Méthode de traduction. Nouvelle édition revue et corrigée. Paris, Didier, 1972.

\section{SOURCES}

Danojlić, Milovan, Pesme. Izbor i pogovor Milan Pražić. Beograd, Nolit-Prosveta-Zavod za udžbenike i nastavna sredstva, 1980

Danojlić, Milovan. Seule la lumière ; poèmes, 1957-2007. Choix et traduction du serbe Vesna Bernard-Radović. Lausanne (Suisse), L’Âge d'Homme, 2010.

\section{O PREVOĐENJU POEZIJE MILOVANA DANOJLIĆA NA FRANCUSKI JEZIK}

U radu analiziramo nekolika poetska ostvarenja Milovana Danojlića iz zbirke „Pesme” (1980) u prevodu na francuski jezik. Ispitivane pesme se nalaze u zbirci „Seule la lumière : poèmes, 1957-2007” (2010) u izboru i prevodu Vesne Bernar-Radović. Sa morfosintaksičkog, semantičkog i stilskog aspekta razmatramo način na koji prevodilac prenosi ideju originala $i$ stil pesnika i utvrđujemo da li uspeva da $u$ ciljni jezik transponuje izvorni ritam pesme i njenu rimu. Istraživanje sprovodimo u sklopu lingvističkog pristupa prevođenju i putem kontrastivnog metoda a za ispitivanje prevodilačkih tehnika primenjenih $u$ procesu prevođenja koristimo klasifikaciju koju je postavio P. Njumark (P. Newmark, „Textbook of Translation", 1988) oslanjajući se na tipologiju Vinea i Darbelnea (J.-P. Vinay et J. Darbelnet, "Stylistique comparée du français et de l'anglais", 1972). Analiziramo tri Danojlićeve pesme: ,Insekti"/,,Les insectes", ,Peršun”/,,Le persil” $i$,,Dunja”/,,Le coing”.

Ključne reči: prevođenje, poezija, prevodilačka tehnika, srpski jezik, francuski jezik 